\title{
Endothelial Function in Children and Adolescents Is Mainly Influenced by Age, Sex and Physical Activity \\ - An Analysis of Reactive Hyperemic Peripheral Artery Tonometry -
}

\author{
Ulrike M. Mueller, MD; Claudia Walther, MD; Jennifer Adam; \\ Kati Fikenzer, MD; Sandra Erbs, MD; Meinhard Mende, PhD; Volker Adams, PhD; \\ Axel Linke, MD; Gerhard Schuler, MD
}

\begin{abstract}
Background: As adolescents rarely experience cardiovascular events, surrogate markers of atherosclerosis are useful to justify and monitor effects of primary prevention and therapy of risk factors. Endothelial function assessed by reactive hyperemic peripheral arterial tonometry (RH-PAT) resulting in a reactive hyperemic index $(\mathrm{RHI})$ is a noninvasive method with limited data for use in children and adolescents.
\end{abstract}

Methods and Results: We performed a total of $931 \mathrm{RHI}$ measurements in 445 high-school students, aged 10-17 years, over a time period of 5 years. Students were randomized by class to $60 \mathrm{~min}$ physical exercise (PE) at school daily (intervention group), or 2 units of 45-min PE weekly (control group). To characterize the factors influencing the RHI, anthropometry, cardiopulmonary exercise testing, blood cholesterol and quality of life were assessed and used to build mixed linear models. Main influential factors were age, with an increase of $\mathrm{RHI}$ from $1.53 \pm 0.42$ in the youngest to $1.96 \pm 0.59$ in the oldest students, sex, with higher values in girls, and physical activity. This increase adjusted by age and sex was estimated as $0.11[0.08,0.14]$ per year. RHI was higher in the intervention group by $0.09[-0.05,0.23]$ in comparison with the control group.

Conclusions: If RH-PAT is used in research or as a clinical tool in adolescents, the shown age- and sex-dependence of RHI have to be taken in account.

Key Words: Adolescents; Endothelial function; Physical exercise; Primary prevention; Reactive hyperemic index

A therosclerosis starts early in life if the currently known cardiovascular risk factors are present. Postmortem studies have demonstrated the presence of atherosclerotic lesions from as early as the first decade of life. ${ }^{1}$ As children rarely experience cardiovascular events, surrogate markers of cardiovascular disease are needed to provide additional risk stratification, and to justify and monitor effects of prevention and therapy of risk factors. ${ }^{2,3}$ A standard evaluation of endothelial function uses brachial artery reactivity measurements such as flow-mediated dilatation (FMD) ${ }^{4,5}$ however, this technique requires much experience and is operator dependent. ${ }^{6}$ Assessment of endothelial (dys-)function using reactive hyperemic peripheral arterial tonometry (RH-PAT) has shown excellent correlation with measures of coronary and peripheral endothelial dysfunction in adults. ${ }^{7,8}$ It provides clinicians with a reliable and reproducible index (the reactive hyper-

\section{Editorial p 637}

emic index (RHI)) of endothelial function in a 15-min test. ${ }^{9}$

In adults, an RHI $<1.35$ indicates impaired endothelial function. ${ }^{7}$ Data providing normal values for RHI in children and adolescents are limited. However, the method is used frequently for study purposes, usually to compare endothelial function in children with cardiovascular risk factors against healthy subjects., ${ }^{2,3}$ Additionally, interventional studies investigating the effect of physical exercise (PE) on RHI in adolescents are lacking.

Thus, using RH-PAT in a large cohort of healthy school children, we aimed to explore the factors influencing the RHI under particular consideration of PE at school.

Received October 2, 2016; revised manuscript received December 27, 2016; accepted January 5, 2017; released online February 11, 2017 Time for primary review: 18 days

Department of Internal Medicine/Cardiology, University Leipzig - Heart Center, Leipzig (U.M.M., C.W., J.A., K.F., S.E., V.A., A.L., G.S.); Department of Cardiology, Kerckhoff Heart Center, Bad Nauheim (C.W.); and Clinical Trial Center, University Leipzig, Leipzig (M.M.), Germany

Mailing address: Ulrike Maria Mueller, MD, Department of Internal Medicine/Cardiology, University of Leipzig - Heart Center, Strümpellstr. 39, 04289 Leipzig, Germany. E-mail: ulrike.mueller@helios-kliniken.de

ISSN-1346-9843 All rights are reserved to the Japanese Circulation Society. For permissions, please e-mail: cj@j-circ.or.jp 


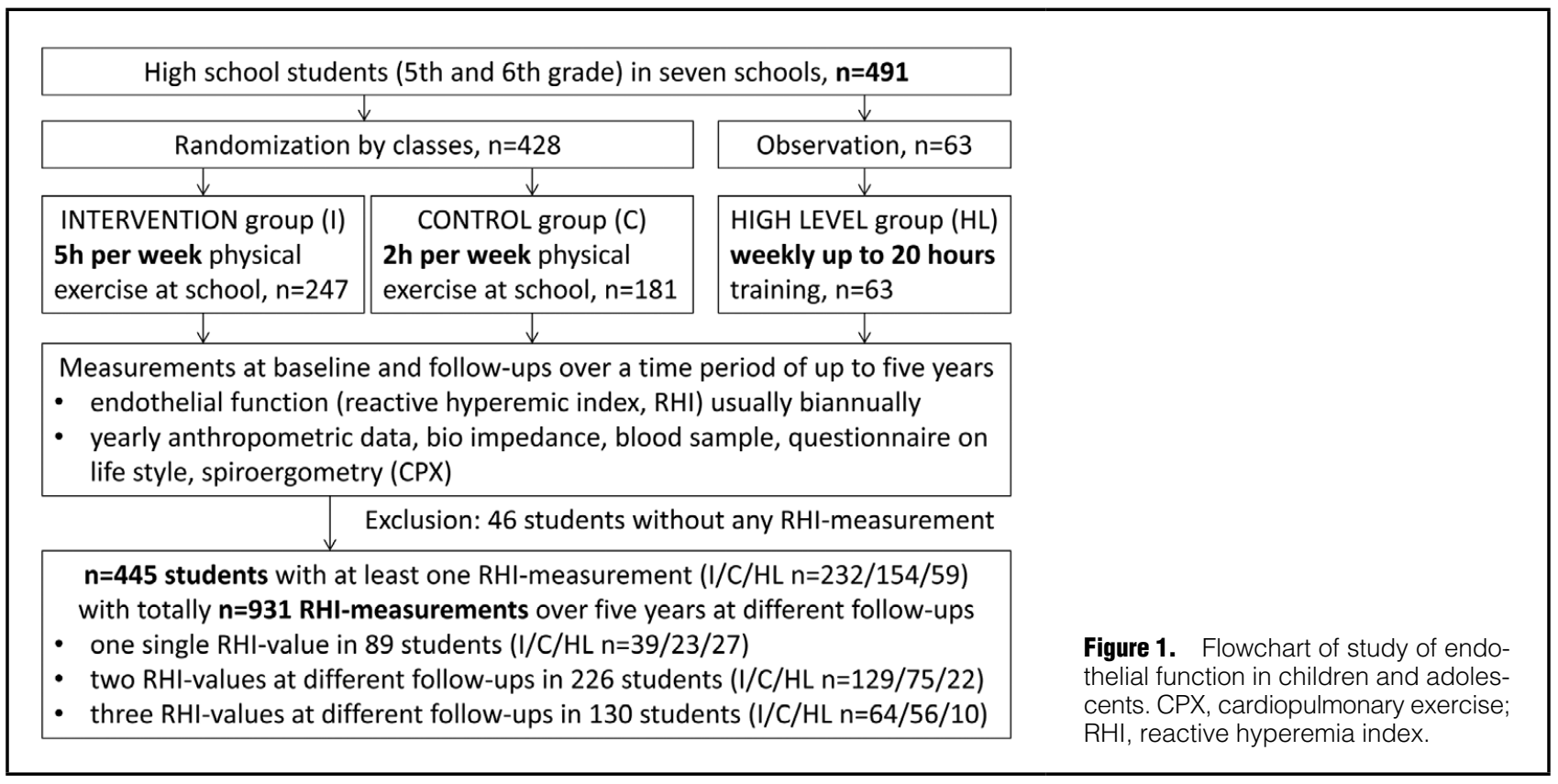

\section{Methods}

\section{Study Design}

Children and adolescents were recruited within the "Leipzig School Project" (clinical trial registration: http://www. clinicaltrials.gov, Identifier: NCT00176371). The study protocol and procedures were approved by the Ethics Review Board at the University of Leipzig. The study was performed in accordance with the ethical standards laid down in the 1964 Declaration of Helsinki. All participants were younger than 18 years at the beginning of the study, so they gave verbal assent and parents gave written informed consent to participate in the study protocol.

A detailed study design was published previously..$^{10,11}$ In brief, the "Leipzig School Project" was a class-wise cluster randomized intervention study designed to investigate the influence of a 60-min PE session daily at school on cardiovascular risk factors in the intervention group in comparison with the otherwise standard of $2 \mathrm{PE}$ units of $45 \mathrm{~min}$ weekly in the control group. At participating schools, 5th and 6 th graders were randomized by class to the intervention or control group. A third study group was a pure observational group in a sports school and consisted of sports students (high-level group) who frequently participated in competitive sporting events, thus representing a maximum of physical fitness attainable under reasonable conditions in school-age children. After baseline assessment, we saw all groups yearly for control measurements.

This subanalysis focusses on the measurement of endothelial function using RH-PAT for assessment of RHI.

\section{Study Subjects}

In 7 schools in the area of Leipzig and Chemnitz, Saxony, Germany, 18 classes with 491 students in grades 5 or 6 were invited to participate in this open-ended, controlled, randomized, school-based exercise program. The program started in 2005, inclusion time was until 2008, and last follow-ups were completed in 2013.

To answer our questions concerning RHI in children, a set of all pupils having at least 1 RHI measurement $(n=445)$ was used. For logistic reasons, it was not possible to perform RH-PAT in all students at each yearly visit, but usually biannually, so the yearly analyses contain different students. The study flowchart is shown in Figure $\mathbf{1}$ and the baseline characteristics of the participating students are given in Table 1.

\section{Measurements}

Procedures were identical in the intervention, control and high-level groups and consisted of RH-PAT, anthropometric data, body composition, questionnaire, blood sample, and cardiopulmonary treadmill exercise test (CPX).

\section{RH-PAT}

RH-PAT was performed using the EndoPAT ${ }^{\circledR}$ device (Itamar Medical Ltd., Caesarea, Israel). Measurements were performed in thermo-neutral quiet surroundings with students positioned supine, following an overnight fast. Fingertip probes were placed on both index fingers and pulse wave amplitudes (PWA) were recorded. After $5 \mathrm{~min}$ of baseline measurement arterial flow in the non-dominant arm was occluded using a blood pressure (BP) cuff inflated to $30 \mathrm{mmHg}$ above systolic pressure. After $5 \mathrm{~min}$ of occlusion, the cuff was rapidly deflated to allow for reactive hyperemia. PWA were recorded for a further $5 \mathrm{~min}$ after the cuff was deflated. An integrated software program calculated the RHI as the ratio of the average PWA measured over $60 \mathrm{~s}$ starting $1 \mathrm{~min}$ after cuff deflation to the average PWA measured at baseline. This ratio is normalized to the concurrent signal from the contralateral finger to correct for changes in systemic vascular tone. Lower RHIs are reflective of greater endothelial dysfunction and risk for atherosclerosis. Details of RH-PAT have been published elsewhere. ${ }^{12,13}$

As mentioned before, it was not possible to perform RHI measurements in all students at each yearly visit for the following reasons: follow-up was performed all day, but RHI measurements only in the morning to ensure 


\begin{tabular}{|c|c|c|c|c|c|c|}
\hline Characteristic & Baseline & $\begin{array}{c}\text { 1st } \\
\text { follow-up }\end{array}$ & $\begin{array}{l}\text { 2nd } \\
\text { follow-up }\end{array}$ & $\begin{array}{c}\text { 3rd } \\
\text { follow-up }\end{array}$ & $\begin{array}{c}\text { 4th } \\
\text { follow-up }\end{array}$ & $\begin{array}{l}\text { 5th } \\
\text { follow-up }\end{array}$ \\
\hline $\mathrm{n}$ & 154 & 271 & 146 & 187 & 112 & 61 \\
\hline I/C/HL group, n (\%) & $\begin{array}{c}84(55) / 56(36) / \\
14(9)\end{array}$ & $\begin{array}{c}152(56) / 90(33) / \\
29(11)\end{array}$ & $\begin{array}{c}61(42) / 57(39) / \\
28(19)\end{array}$ & $\begin{array}{c}107(57) / 70(37) / \\
10(5)\end{array}$ & $\begin{array}{c}52(46) / 41(36) / \\
19(17)\end{array}$ & $\begin{array}{c}33(54) / 27(44) / \\
1(2)\end{array}$ \\
\hline Female in $\mathrm{I} / \mathrm{C} / \mathrm{HL}, \mathrm{n}(\%)$ & $\begin{array}{c}37(44) / 20(36) / \\
6(43)\end{array}$ & $\begin{array}{c}75(49) / 51(57) / \\
12(41)\end{array}$ & $\begin{array}{c}28(46) / 21(37) / \\
7(25)\end{array}$ & $\begin{array}{c}46(43) / 39(56) / \\
5(50)\end{array}$ & $\begin{array}{c}26(50) / 18(44) / \\
4(21)\end{array}$ & $\begin{array}{c}15(45) / 17(63) / \\
0(0)\end{array}$ \\
\hline $\mathrm{RHI}$ & $1.53 \pm 0.42$ & $1.49 \pm 0.45$ & $1.58 \pm 0.50$ & $1.71 \pm 0.49$ & $1.97 \pm 0.66$ & $1.96 \pm 0.59$ \\
\hline $\mathrm{RHI}<1.35, \mathrm{n}(\%)$ & $72(46.8)$ & $153(56.6)$ & $61(41.8)$ & $53(28.3)$ & $17(15.2)$ & $9(14.8)$ \\
\hline Age (years) & $11.66 \pm 0.93$ & $12.44 \pm 0.67$ & $13.17 \pm 0.77$ & $14.38 \pm 0.68$ & $15.23 \pm 0.76$ & $16.47 \pm 0.46$ \\
\hline Height (cm) & $151.24 \pm 8.99$ & $156.34 \pm 8.11$ & $161.82 \pm 8.98$ & $166.89 \pm 8.72$ & $170.61 \pm 8.73$ & $172.59 \pm 10.62$ \\
\hline Body weight (kg) & $43.79 \pm 10.18$ & $45.93 \pm 9.62$ & $51.20 \pm 11.13$ & $57.05 \pm 9.68$ & $61.65 \pm 11.99$ & $63.83 \pm 9.91$ \\
\hline $\mathrm{BMI}\left(\mathrm{kg} / \mathrm{m}^{2}\right)$ & $18.93 \pm 3.02$ & $18.66 \pm 2.9$ & $19.38 \pm 2.95$ & $20.42 \pm 2.71$ & $21.04 \pm 3.07$ & $21.37 \pm 2.26$ \\
\hline BMI percentile & $55.76 \pm 28.54$ & $47.44 \pm 28.93$ & $49.73 \pm 28.39$ & $53.91 \pm 25.73$ & $54.26 \pm 28.65$ & $53.66 \pm 24.69$ \\
\hline $\begin{array}{l}\text { BMI >90th percentile, } \\
n(\%)\end{array}$ & $45(10.5)$ & $41(9.6)$ & $40(10.3)$ & $40(11.4)$ & $24(9.4)$ & $7(9.0)$ \\
\hline BMI-SDS & $0.20 \pm 0.94$ & $-0.10 \pm 1.02$ & $0.01 \pm 0.95$ & $0.13 \pm 0.86$ & $0.15 \pm 0.96$ & $0.12 \pm 0.77$ \\
\hline Heart rate at rest $(1 / \mathrm{min})$ & $79 \pm 10.82$ & $96 \pm 14$ & $83 \pm 19$ & $72 \pm 10$ & $72 \pm 12$ & $70 \pm 16$ \\
\hline $\mathrm{SBP}(\mathrm{mmHg})$ & $99 \pm 10$ & $102 \pm 11$ & $108 \pm 12$ & $99 \pm 9$ & $107 \pm 11$ & $97 \pm 8$ \\
\hline $\mathrm{DBP}(\mathrm{mmHg})$ & $63 \pm 7$ & $70 \pm 8$ & $69 \pm 10$ & $62 \pm 8$ & $62 \pm 8$ & $60 \pm 3$ \\
\hline $\begin{array}{l}\text { Fat-free mass ( } \% \text { body } \\
\text { weight) }\end{array}$ & $79.28 \pm 6.99$ & $80.55 \pm 7.03$ & $81.20 \pm 6.87$ & $78.99 \pm 6.99$ & $78.92 \pm 8.32$ & $78.58 \pm 7.91$ \\
\hline $\begin{array}{l}\text { Muscle mass (\% body } \\
\text { weight) }\end{array}$ & $48.21 \pm 5.45$ & $48.96 \pm 5.78$ & $50.46 \pm 5.82$ & $50.18 \pm 5.94$ & $50.96 \pm 6.82$ & $51.56 \pm 6.52$ \\
\hline $\begin{array}{l}\text { Heart rate at maximum } \\
(1 / \mathrm{min})\end{array}$ & $204 \pm 10$ & $204 \pm 10$ & $203 \pm 10$ & $199 \pm 7$ & $201 \pm 8$ & $197 \pm 6$ \\
\hline$\dot{\mathrm{V}} \mathrm{O}_{2 \text { peak }}(\mathrm{mL} / \mathrm{min} / \mathrm{kg})$ & $50.86 \pm 9.68$ & $48.21 \pm 8.72$ & $53.69 \pm 8.84$ & $48.78 \pm 8.58$ & $53.86 \pm 10.02$ & $47.31 \pm 9.28$ \\
\hline Maximum power (W) & $132.78 \pm 35.71$ & $155.89 \pm 36.58$ & $181.08 \pm 46.72$ & $187.61 \pm 51.84$ & $209.19 \pm 63.35$ & $172.50 \pm 53.15$ \\
\hline VE peak (L/min) & $78.25 \pm 17.93$ & $80.28 \pm 17.16$ & $96.94 \pm 25.37$ & $95.10 \pm 22.86$ & $118.03 \pm 31.08$ & $105.35 \pm 26.96$ \\
\hline Time to max. effort (s) & $1,010.96 \pm 91.90$ & $1,083.97 \pm 95.84$ & $1,101.88 \pm 113.14$ & $1,077.66 \pm 101.87$ & $1,099.31 \pm 109.62$ & $1,097.24 \pm 108.98$ \\
\hline Motor quotient & $95.65 \pm 16.94$ & $112.77 \pm 13.85$ & $108.55 \pm 16.61$ & $116.62 \pm 11.50$ & $123.00 \pm 7.21$ & $121.00 \pm 5.66$ \\
\hline Cholesterol (mmol/L) & $4.21 \pm 0.69$ & $4.17 \pm 0.64$ & $4.14 \pm 0.67$ & $3.88 \pm 0.66$ & $3.93 \pm 0.77$ & $3.79 \pm 0.80$ \\
\hline $\mathrm{HDL}(\mathrm{mmol} / \mathrm{l})$ & $1.41 \pm 0.33$ & $1.44 \pm 0.33$ & $1.48 \pm 0.33$ & $1.41 \pm 0.32$ & $1.36 \pm 0.32$ & $1.42 \pm 0.29$ \\
\hline LDL (mmol/L) & $2.21 \pm 0.57$ & $2.18 \pm 0.56$ & $2.23 \pm 0.59$ & $2.19 \pm 0.56$ & $2.08 \pm 0.58$ & $2.00 \pm 0.59$ \\
\hline $\mathrm{TG}(\mathrm{mmol} / \mathrm{L})$ & $1.09 \pm 0.57$ & $1.08 \pm 0.66$ & $1.07 \pm 0.55$ & $0.98 \pm 0.50$ & $0.99 \pm 0.52$ & $1.00 \pm 0.54$ \\
\hline Leisure activity index & $3.40 \pm 0.67$ & $3.73 \pm 0.57$ & $3.58 \pm 0.57$ & $3.59 \pm 0.58$ & $3.58 \pm 0.66$ & $3.67 \pm 0.54$ \\
\hline Sports index & $3.18 \pm 0.59$ & $3.35 \pm 0.61$ & $3.31 \pm 0.71$ & $3.19 \pm 0.69$ & $3.33 \pm 0.71$ & $3.25 \pm 0.66$ \\
\hline Life quality index & $3.49 \pm 0.50$ & $3.96 \pm 0.48$ & $3.85 \pm 0.46$ & $3.78 \pm 0.47$ & $3.86 \pm 0.51$ & $3.80 \pm 0.48$ \\
\hline
\end{tabular}

This substudy focused on RHI measurements. The yearly measurements contain different students, as RHI measurements were not performed yearly for each student. Data are mean \pm standard deviation unless otherwise indicated. BMI, body mass index; C, Control group; DBP, diastolic blood pressure; HDL, high-density lipoprotein; HL, High-level group; I, Intervention group; LDL, low-density lipoprotein; MET, metabolic equivalent of task; $\mathrm{RHI}$, reactive hyperemic index; SBP, systolic blood pressure; TG, triglycerides; $\mathrm{V}^{\mathrm{E}}$, expiratory volume; $\mathrm{V}_{2 p e a k}$ peak oxygen uptake at spiroergometry (cardiopulmonary exercise).

overnight fast; in the first 2 study years the technique was not available at our study center.

\section{Anthropometry, Body Composition, Questionnaire and Blood Samples}

Height, body weight and BP were measured with standard medical devices. The body mass index (BMI) was calculated as the quotient of weight in $\mathrm{kg}$ divided by the square of height in meters. Age- and sex-adjusted BMI percentiles and standard deviation score (BMI-SDS) were determined by using age- and sex-adjusted curves for children and adolescents. ${ }^{14}$

Students were asked to attend the RH-PAT and blood tests after fasting. We assessed blood count, glucose, lowdensity lipoprotein-cholesterol, high-density lipoproteincholesterol, and triglycerides. Body composition [fat-free mass (FFM) and muscle mass (MM)] was assessed by bioimpedance (Akern, Italy).

All participants had to complete a questionnaire containing 13 items that evaluated quality of life, and physical activity in general and in leisure time. From these items 2 indices, activity index in general, and leisure index for leisure physical activity, were calculated. To assess family history all parents were asked at baseline to complete a questionnaire on cardiovascular risk factors, including obesity (height and weight were requested), diabetes, arterial hypertension, smoking, hyperlipidemia, and coronary artery disease. We investigated whether the known cardiovascular family risk factors were associated with RHI in children: (1) if one or both parents had at least one risk factor; and (2) if the total number of parental risk factors in mothers, fathers or both parents showed an association with RHI in children. 


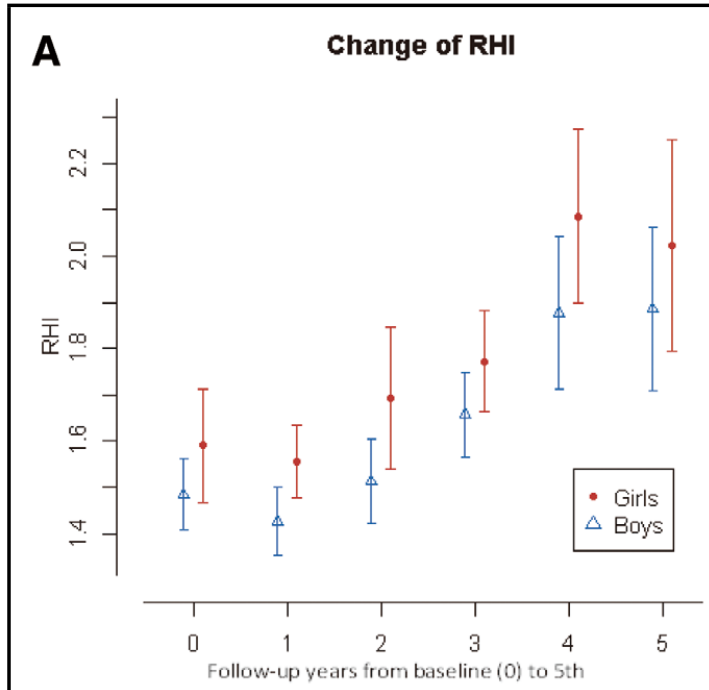

B

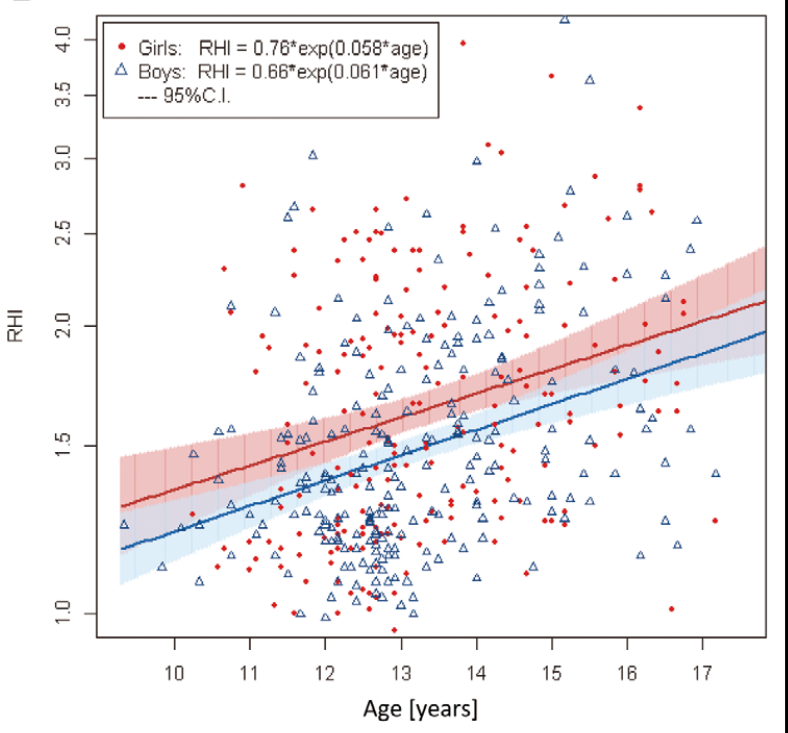

Figure 2. Sex (A) and age (B) dependency of reactive hyperemia index $(\mathrm{RHI})$ in children and adolescents.

\section{CPX}

To assess fitness, participants underwent a graded treadmill exercise test (Woodway USA, Inc., WI, USA) with spirometry until exhaustion, according to a modified Bruce protocol for children, starting at $1.7 \mathrm{mph}$ and 0 degrees. Oxygen uptake $\left(\dot{\mathrm{VO}}_{2}\right)$ was recorded using a portable spirometry system (K4b2, Cosmed Srl., Italy). Workload was increased every $3 \mathrm{~min}$. At the end of each level the subjects had to indicate their personal perception of exhaustion according to the Borg rating on a scale from 1 to 10 ( $1=$ no exhaustion, $10=$ maximal exhaustion $)$, and cardiopulmonary indicators were recorded [BP, heart rate (HR), $\dot{\mathrm{V} O}$, expiratory volume ( $\dot{\mathrm{VE}})$, respiratory exchange ratio (RER, ratio of carbon dioxide production to $\dot{\mathrm{VO}}_{2}$ )] Requirements to ensure that subjects reached their maximum effort with this protocol included at least 2 of the following: maximal HR > 200 beats/min, RER >1.1, a plateau in oxygen consumption, and subjective Borg rating

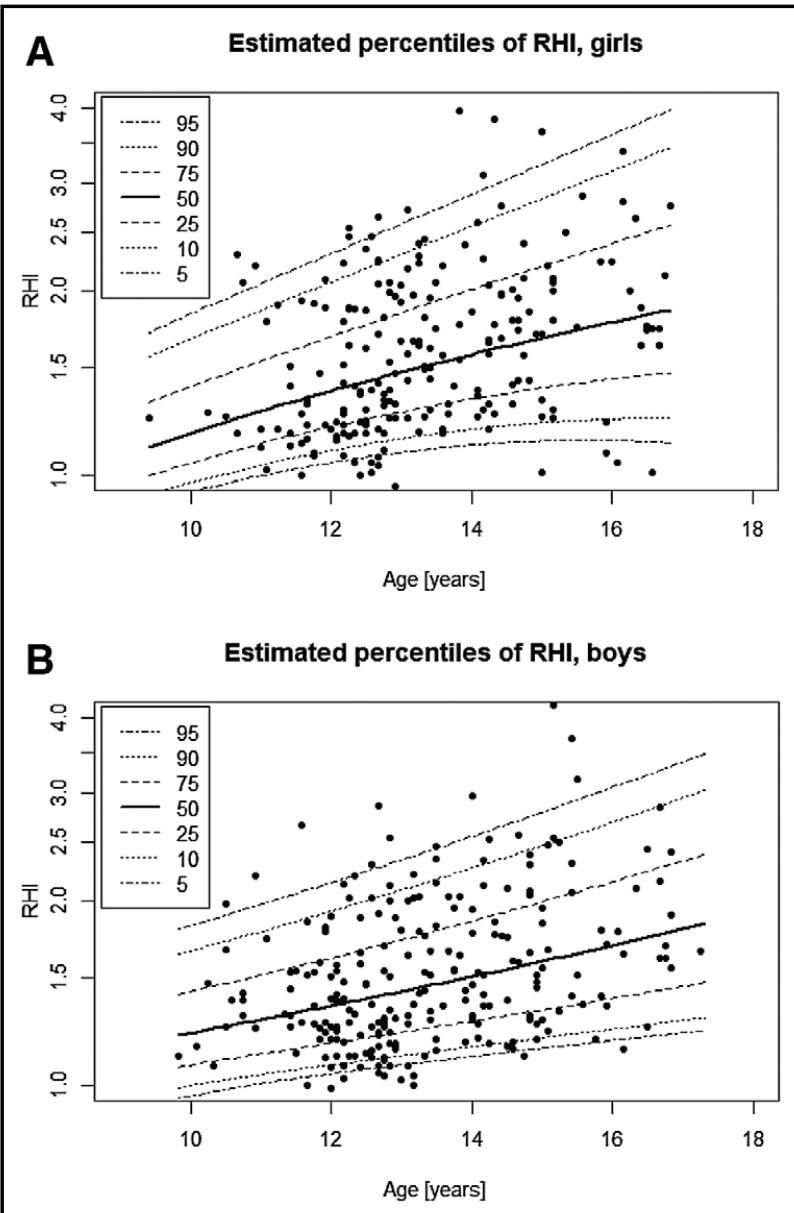

Figure 3. Estimated percentiles of reactive hyperemia index $(\mathrm{RHI})$ in $(\mathbf{A})$ girls and $(\mathbf{B})$ boys.

of $10 . \mathrm{VO}_{2}$ was calculated in relation to body weight and is given in $\mathrm{mL} / \mathrm{min} / \mathrm{kg}$.

All students and parents were given the test results of anthropometry and CPX with a rating on health status and performance.

\section{Endpoints}

The primary endpoint in this substudy was the RHI. Variables analyzed as secondary endpoints were parameters of $\mathrm{CPX}\left[\dot{\mathrm{VO}}_{2 \text { peak, }} \mathrm{HR}\right.$ at rest and at maximum, maximum running duration (Tmax) and peak power], anthropometric values and body composition.

\section{Statistical Analysis}

Data preparation and descriptive statistics were performed by IBM SPSS Statistics version 23, and R (packages moments, gamlss, ggplot2, lme4, Hmisc) for advanced statistics.

The study cohorts were characterized by standard descriptive statistics. Because missing status of RHI values does not depend on the RHI values themselves, the assumption "missing completely at random" is plausible, which means that complete case analyses give unbiased results.

We compared the RHI of normal and overweight/obese children per year by paired t-test. Most analyses were 


\begin{tabular}{|c|c|c|c|c|c|c|}
\hline & \multicolumn{2}{|c|}{ RHI } & \multicolumn{2}{|c|}{ Age } & \multicolumn{2}{|c|}{$\dot{\mathrm{VO}}_{2 \text { peak }}$} \\
\hline & Boys & Girls & Boys & Girls & Boys & Girls \\
\hline \multicolumn{7}{|l|}{ Baseline } \\
\hline Mean & 1.48 & 1.59 & 11.6 & 11.6 & 46.9 & 41.9 \\
\hline $95 \% \mathrm{Cl}$ & $1.41,1.56$ & $1.47,1.71$ & $11.5,11.7$ & $11.5,11.8$ & $45.5,48.3$ & $40.7,43.1$ \\
\hline \multicolumn{7}{|c|}{1 st follow-up } \\
\hline Mean & 1.43 & 1.56 & 12.3 & 12.4 & 51.6 & 44.6 \\
\hline $95 \% \mathrm{Cl}$ & $1.35,1.50$ & $1.48,1.63$ & $12.3,12.4$ & $12.3,12.4$ & $50.4,52.7$ & $43.4,45.8$ \\
\hline \multicolumn{7}{|c|}{ 2nd follow-up } \\
\hline Mean & 1.51 & 1.69 & 13.4 & 13.4 & 53.4 & 43.9 \\
\hline $95 \% \mathrm{Cl}$ & $1.42,1.61$ & $1.54,1.84$ & $13.3,13.4$ & $13.3,13.6$ & $52.2,54.6$ & $42.7,45.2$ \\
\hline \multicolumn{7}{|c|}{ 3rd follow-up } \\
\hline Mean & 1.66 & 1.77 & 14.3 & 14.4 & 55.1 & 43.8 \\
\hline $95 \% \mathrm{Cl}$ & $1.57,1.75$ & $1.66,1.88$ & $14.2,14.4$ & $14.3,14.5$ & $53.9,56.4$ & $42.7,45.0$ \\
\hline \multicolumn{7}{|c|}{ 4th follow-up } \\
\hline Mean & 1.88 & 2.09 & 15.3 & 15.2 & 55.7 & 42.3 \\
\hline $95 \% \mathrm{Cl}$ & $1.71,2.04$ & $1.90,2.27$ & $15.1,15.4$ & $15.0,15.4$ & $54.2,57.2$ & $40.9,43.7$ \\
\hline \multicolumn{7}{|c|}{ 5th follow-up } \\
\hline Mean & 1.89 & 2.02 & 16.5 & 16.3 & 58.2 & 41.5 \\
\hline $95 \% \mathrm{Cl}$ & $1.71,2.06$ & $1.79,2.25$ & $16.3,16.7$ & $16.1,16.5$ & $55.9,60.4$ & $39.9,43.1$ \\
\hline
\end{tabular}

$\mathrm{Cl}$, confidence interval. Other abbreviations as in Table 1.

performed applying linear mixed-effects models (LMM). ${ }^{15}$ This tool is able to adjust for age and sex, and to model correlation structures of repeated measurements and clustered data. Additionally, LMM are able to deal with missing values. We considered models with group and sex as fixed factors, age, BMI and $\dot{\mathrm{V}}_{2 \text { peak }}$ as continuous covariates, and subject and class as factors with random intercepts. We checked for possible interactions, but removed the interaction terms if no significant improvement was seen. Finally, we applied LMM to look for variables possibly multiply associated with the RHI. To do so, we started with a full model (24 variables) and reduced step by step to get a sparse model with significant variables. Fixed effects were estimated, and $95 \%$ confidence intervals were calculated by profile likelihood.

To create percentile plots, a set of independent values had to be created. Thus, exactly 1 RHI per student was randomly chosen. Percentiles were calculated by the $\mathrm{R}$ package gamlss and percentile curves were plotted. ${ }^{16}$ Positive skewness and kurtosis $>4$ restricted the number of possible functions. The aim was to fit relatively smooth curves with low total deviation, good agreement between observed and fitted percentiles, and without artefacts.

Significance level for two-sided tests was generally $5 \%$.

\section{Results}

\section{Study Population}

Baseline and follow-up parameters are given in Table 1. The analyses containing all $931 \mathrm{RHI}$ were comparable with the calculations when only the longitudinal data of the 130 students with 3 RHI measurements over the study time were used. Therefore, the following results include all 931 measured RHI.

\section{RHI, Age and Sex}

RHI increased significantly with increasing age (Table 1,
Figures 2,3). The mean RHI of girls increased from 1.59 $[1.47,1.71]$ in the youngest cohort at baseline to $1.69[1.54$, $1.84]$ after 2 , and $2.09[1.90,2.27]$ after 4 years; the average RHI of boys increased from $1.48[1.41,1.56]$ to 1.51 [1.42, $1.61]$ and $1.88[1.71,2.04]$ after 2 and 4 years, respectively. Most students had higher RHI in the follow-up in comparison with baseline. This increase adjusted by age and sex was estimated as $0.11[0.084,0.14]$ per year and was significant. The RHI for boys and girls by year are given in Table 2.

At all time-points the RHI was higher in girls than in boys (Figures 2,3). The mean difference was 0.11 [ -0.002 , 0.23], which became significant after adjustment for BMI percentile or intervention group (Table 3B,C).

Based on our data we calculated reference values for boys and girls (Table S1, Figure 3).

\section{RHI and Intervention or Sports Performance}

Regular physical activity had an effect on RHI. Students in the intervention group had RHI that were higher than those of the control group by 0.09 [ $-0.052,0.23]$, adjusted for age and sex. The annual difference between the highlevel and control groups was 0.14 [-0.05, 0.33] (Table 3).

On the other hand, a dependency of $\mathrm{VO}_{2 \text { peak }}$ after adjustment for age and sex was hardly seen at all $(0.0023$ per $10 \mathrm{~mL} / \mathrm{min} / \mathrm{kg}[-0.022,0.068])$.

\section{RHI and BMI}

We observed a weak association of BMI percentiles and RHI (Table 3): an increase of BMI percentile by 10 was associated with an increase in RHI by 0.012 [0.000, 0.024]. However, a difference of mean RHI between children with normal BMI and those who were overweight/obese could not be proven.

\section{Variables Possibly Associated With RHI}

We looked for a possible association between 24 variables 
Table 3. Linear Mixed Models for Association With RHI

\begin{tabular}{|c|c|c|}
\hline & Coefficient & $95 \% \mathrm{Cl}$ \\
\hline \multicolumn{3}{|c|}{ A) Adjustment for sex and age } \\
\hline Constant & 1.33 & $1.23,1.43$ \\
\hline Age (years) & 0.111 & $0.084,0.137$ \\
\hline Girl & 0.114 & $-0.002,0.230$ \\
\hline \multicolumn{3}{|c|}{ B) Possible influence of intervention } \\
\hline Constant & 1.26 & $1.14,1.39$ \\
\hline Age (years) & 0.111 & $0.090,0.131$ \\
\hline Girl & 0.117 & $0.046,0.189$ \\
\hline \multicolumn{3}{|l|}{ Intervention } \\
\hline I group & 0.090 & $-0.052,0.233$ \\
\hline HL group & 0.141 & $-0.050,0.332$ \\
\hline \multicolumn{3}{|c|}{ C) Dependence from BMI percentile } \\
\hline Constant & 1.27 & $1.17,1.38$ \\
\hline Age (years) & 0.108 & $0.087,0.129$ \\
\hline Girl & 0.112 & $0.042,0.183$ \\
\hline BMI percentile (per 10) & 0.012 & $0.000,0.024$ \\
\hline \multicolumn{3}{|c|}{ D) Possible association of $\mathrm{RHI}$ with $\mathrm{V}_{2 \text { peak }}$} \\
\hline Constant & 1.21 & $0.95,1.47$ \\
\hline Age (years) & 0.109 & $0.088,0.130$ \\
\hline Girl & 0.142 & $0.061,0.225$ \\
\hline$\dot{\mathrm{VO}}_{2 \text { peak }}($ per $10 \mathrm{ml} / \mathrm{min} / \mathrm{kg})$ & 0.0023 & $-0.022,0.068$ \\
\hline \multicolumn{3}{|c|}{ E) With RHI-associated variables (baseline and follow-up) } \\
\hline Constant & 1.84 & $1.59,2.11$ \\
\hline Age & 0.103 & $0.082,0.125$ \\
\hline Girl & 0.124 & $0.051,0.196$ \\
\hline BMI percentile (per 10) & 0.017 & $0.005,0.030$ \\
\hline DBP at rest (per 10) & -0.090 & $-0.126,-0.055$ \\
\hline
\end{tabular}

Intervention group had $1 \mathrm{~h}$ daily of physical exercise in school in contrast to the control group with $2 \times 45$-min units per week. High-level group contains athletes with regular intensive training sessions up to $20 \mathrm{~h}$ weekly. Abbreviations as in Tables 1,2.

and RHI. Potential candidates were, for example, age, sex, year of measurement, BMI, FFM and MM, family history, $\mathrm{HR}$, systolic and diastolic BP at rest, sports and leisure time index, as well as $\dot{\mathrm{V}} \mathrm{O}_{2 \text { peak, }}$ maximal running time, and maximal power. We found a model with age, sex, BMI percentile and diastolic $\mathrm{BP}$ at rest. The correlation of the first 3 factors has already described. Additionally, an increase in diastolic BP by $10 \mathrm{mmHg}$ was associated with a decrease in RHI by $-0.09[-0.13,-0.05]$. Family history was assessed at baseline by self-reported questionnaire, but parental information was only available from approximately $55 \%$ of the families (most of them did not fill out the questionnaires or the data were incomplete). With the limited and possibly biased data, associations between cardiovascular risk factors in the parents and the RHI in adolescents could not be proved. Additionally, the calculated "effect" of parental risk factors was small compared with the effects of age and sex. The highest association was found if risk factors were present in both parents, with reduction in RHI by -0.073 $[-0.191 ; 0.045]$. Maternal risk factors, especially smoking, had more influence than paternal ones on reducing the RHI, but also without reaching significance, and with a wide confidence interval including 0 .

\section{Discussion}

The purpose of this study was to assess the factors influencing the RHI in healthy children and adolescents with a particular focus on the effect of PE at school.

First, a fundamental comment: there was a very wide intra- and interindividual variation in RHI (Figure 2B). We found some associations, but are far from knowing the causal relationships.

Interestingly, we found an interaction between RHI, age and sex. Additionally, we measured a nonsignificantly higher RHI in our intervention group with daily PE lessons and even higher in the high-level group. In contrast, RHI was not directly dependent on fitness measured by $\dot{\mathrm{V}}_{2}$ peak. Other influential factors were a weak association of BMI percentile and RHI, and an inverse association between diastolic BP and RHI.

\section{Use of RH-PAT in Children and Adolescents}

We used the EndoPAT ${ }^{\circledR}$ device to assess endothelial function, which focuses on small resistance vessels to evaluate endothelium-dependent flow-mediated dilation. This dynamic testing method enables evaluation of the endothelial cell response to mechanical stress to test for the presence of endothelial dysfunction as a systemic disorder.

As with other studies, we agree that RH-PAT is a promising noninvasive technique to evaluate endothelial function and was well tolerated by all the children. None of the RHPAT-tests had to be terminated early, and all measurements were analyzable. Further advantage of the measurement of 
RHI using EndoPat ${ }^{\circledR}$ is that it is simple to use and needs less training than FMD. ${ }^{13}$

\section{Factors Associated With RHI: Clinical Aspects}

Endothelial dysfunction is present in children with risk factors for atherosclerosis, such as hypercholesterolemia, obesity, smoking or diabetes, before anatomical evidence of plaque formation in the arteries studied. ${ }^{17}$ As endothelial dysfunction is believed to be a pan-systemic disease associated with numerous disease states and shown to respond well to treatment even in childhood, lifestyle modification may augment RHI. The higher RHI values that we found in the intervention group and high-level group may also account for this. As we have shown a positive effect of daily PE lessons on $\dot{\mathrm{V}} \mathrm{O}_{2 \text { peak }}$ in the first 2 years of our study, ${ }^{11,18}$ but less after 4 years of intervention, ${ }^{10}$ it could be argued that the positive effect of PE on endothelial function lasts longer than on cardiovascular performance. This underlines the necessity of lifelong regular physical activity to prevent cardiovascular diseases.

Another important and interesting finding of our study was the sex difference for the RHI in all age groups. Associations between pubertal development and RHI, and between age, sex, and RHI in children and adolescents have been reported in smaller cohorts. ${ }^{19-21}$ A possible effect of sex hormones could explain this difference even in our youngest study group because of the earlier puberty of girls, as estrogens might protect the different vascular wall layers against early changes. Additionally, it has to be kept in mind that this cohort of adolescents was mainly healthy. Thus, the traditional cardiovascular risk factors will have minimal effect on vascular features.

As with other studies, we observed high variability in the RHI values. ${ }^{22}$ Haller et al described a wide range of RHI in their cohort of children with type 1 diabetes (age 14.6 \pm 2.7 years): RHI was $1.63 \pm 0.5$ when compared with children without diabetes (mean RHI 1.95 $\pm 0.3, \mathrm{P}<0.01$ ). ${ }^{3}$ Our values in the 14-year-olds were higher than in the diabetes group but lower than in the healthy group. As other studies have found comparable data (e.g., male diabetic subjects vs. controls ( $1.60 \pm 0.32$ vs. $1.92 \pm 2.5),{ }^{2}$ we point out the necessity of considering the age and sex of the test persons. Our age range was small $(<0.9$ years) because of class-wise testing. High standard deviations of age in other studies may have a strong effect on RHI. Therefore a lot of unobserved associations (e.g., social, genetic, or adolescent) have to be taken in account as additional influences on the results. In terms of children and adolescents, family history may also be important for the individual's RHI. However, associations between cardiovascular risk factors in the parents and the RHI in children could not be proven in our study, in contrast to the "Cardiovascular risk in Young Finns study", where parental smoking status in childhood had an influence on FMD in young adults. ${ }^{23}$ Because those subjects were much older than our cohort (24-39 years), a direct comparison cannot be made. An investigation of our population 10-20 years later could answer the question whether the small, but not significant, "effects" of parental risk factors in adolescents lead to more endothelial dysfunction in young adulthood. Furthermore, the low rate of response to the questionnaires may have biased our data.

Our findings may also be relevant for the planning of further studies, because the large variability implies the need for larger study sizes to detect effects when using RHPAT for evaluation of endothelial function. ${ }^{22}$

\section{RHI and Other Noninvasive Parameters of Endothelial Function: Are They Comparable?}

Noninvasive measurements for risk stratification include, for example, FMD, intima-media thickness (IMT), carotid artery compliance (CAC), and RH-PAT. In adults, RHPAT has been shown to correlate closely with FMD and IMT, as well as with direct measures of coronary arterial function. ${ }^{7,13}$ From adult studies we know that endothelial function deteriorates with increasing age in otherwise healthy subjects. ${ }^{24}$ This raises the question of why do we see an increase in RHI during adolescence, which seems paradoxical, despite the presence of atherosclerotic lesions in the first decade of life. ${ }^{1}$

The data on the relationships between age, sex, and indices of vascular damage are limited and partly controversial in adolescence as in adulthood. Age-dependency with an increase of RHI in adolescence has been foundpreviously. ${ }^{19}$ Recently, Hopkins et al ${ }^{25}$ determined the relationship between FMD, age, and sex in 978 healthy children and adolescents aged 6-18 years. They observed that FMD depended on brachial artery diameter. However, they found an overall sex and age difference in FMD, mainly driven by changes in FMD from 16 to 18 years between males and females, with the decrease in FMD apparent in postpubertal males, but not in females. Comparable to our RHI data, they reported high variability in FMD. Furthermore, they reported unsteady FMD values throughout the years. It is difficult to explain why FMD at the age of 8 years was higher than at 6 or 10 years, and at 10 years higher than at 7, 14 and 15 years. ${ }^{25}$ However, those authors concluded that age- and sex-specific FMD data are not needed in pediatric populations. In contrast, our data show that age and sex have to be taken into account when analyzing RH-PAT findings in children and adolescents. An important strength of our study is that we provide longitudinal study data not only for RHI in 10- to 17-year-old subjects, but also for 130 students with 3 RHI measurements at different ages, showing comparable ageand sex-related differences of RHI.

In the "Cardiovascular risk in Young Finns" study follow-up, ${ }^{26}$ measurements of FMD, IMT and CAC were performed in healthy young adults aged 24-39 years. An age-dependency of IMT and CAC was found, but no interaction between age and FMD. Additionally, the sex differences in these parameters were mostly explained by differences in risk factors and vessel size; however, FMD was initially higher in women, but after adjustment for baseline brachial artery diameter, men had significantly higher values. ${ }^{26}$ We reiterate that our study population was much younger, which also means that fewer cardiovascular risk factors were present in our subjects. Moreover, depending on the study subjects and study size, other studies dealing with endothelial function show variable results with and without sex and age differences in carotid or femoral IMT. ${ }^{27-30}$ Another strength of our analysis is that, in addition to sex and age, we included many other potential covariates in the LMM, though age and sex remained the most relevant variables with regard to RHI.

Even if RH-PAT is technically easier to perform than FMD, influences on measurement such as stress in the morning, anxiety, clandestine breakfast, passive smoking at home and other factors may have more subtle influences in children and adolescents than in adults, especially on small vessel reactivity. 


\section{Molecular Mechanisms}

For many years, paracrine control of vessels' function has been attributed exclusively to the endothelium. ${ }^{31}$ Endothelium-dependent dilation is influenced by various molecules. Nitric oxide (NO) is the major endotheliumderived relaxing factor; others are prostacyclin and endothelial-derived hyperpolarizing factor (EDHF). ${ }^{32}$ Although $\mathrm{NO}$ affects the macro- and microcirculation, the importance of EDHF increases as the vessel's diameter decreases. ${ }^{33}$ Therefore, EDHF plays a prominent role in the resistance vessels of the microcirculation. ${ }^{34}$ Because of EDHF, microvascular endothelial cells hyperpolarize upon agonist stimulation more effectively than do macrovascular cells. ${ }^{35}$ In addition to the vessel's size and endothelial function, the vasoactive role of adventitial cells and perivascular adipose tissue is now a topic of research. ${ }^{31}$ Different thickness of vessel layers in resistance and conduit vessels, interactions of various concentrations of vasoconstrictive and vasodilative molecules, and interactions between the endothelium, perivascular tissue and smooth muscle cells, may account for some failure of RHI- and FMD-correlation, as they may reflect different aspects of vascular damage. Although different methods are used to noninvasively assess endothelial function, they may not be interchangeable. ${ }^{36} \mathrm{RHI}$ and FMD do not measure the same vascular responses to shear stress induced by 5-min occlusion of the brachial artery. RH-PAT reflects the vasodilator function in the microvasculature of the fingers, whereas FMD considers the vasodilator function in large conduit vessels. Moreover, for both techniques the interaction between vessel wallderived vasodilator and vasoconstrictor factors has to be discussed. We also considered whether or not high-flowmediated constriction prior to vasodilation ${ }^{37}$ may be found more in the finger tips than in the brachial artery, and thus may also have an influence on the relation of FMD and RHI, especially in young study cohorts. Therefore, the correlation between the different vasoactive substances and FMD and RHI remains to be established.

We considered the same concerns raised by Kelly et al:19 that is, the age-dependency of RHI may be a measurement error related to fingertip probes, which have one size for all persons. Some studies therefore favor FMD. However, in a former study we found a close correlation between the RHI in children and the endothelial NO synthase (eNOS)Ser $^{1177}$ phosphorylation $(r=0.66)$ induced by high-density lipoprotein-cholesterol (as it is capable of modifying the production of NO) at the stimulatory site, and the eNOS$\mathrm{Thr}^{495}$ phosphorylation $(\mathrm{r}=-0.60)$ at the inhibitory site. ${ }^{38}$ Therefore, larger studies are needed to clarify the usefulness of the tests, the influence of growth-related changes to vessel function, the relation between both, and the correlation with concentrations of vasoactive molecules.

\section{Study Limitations}

This substudy was, in essence, purely explorative. Yearly measurements of RHI in all participants might have added additional information, but were not possible for several reasons (availability of device, high costs of probes, and subjects tested in the afternoon without RHI).

Because of the hormonal influence on endothelial function in postpubertal girls, tests on endothelial function should be performed in the third week of the menstrual cycle, as the test results of endothelial function are the highest at that time. This was not always feasible. However, it can be supposed that doing so would have increased the measured values in postpubertal girls, and therefore led to a larger difference between boys and girls.

\section{Conclusions}

In adolescence RHI increases with age. Girls have higher values than boys. Daily PE at school may improve endothelial function measured by RH-PAT. If RH-PAT is used in research or as a clinical tool, the age- and sex-dependency and wide variation in results have to be taken in account.

\section{Acknowledgments}

We thank the children and their parents, the teachers and principals of Annenmittelschule Chemnitz, Evangelisches Schulzentrum Leipzig, Johannes-Kepler-Gymnasium Chemnitz, Mittelschule and Gymnasium Brandis, Sportgymnasien Chemnitz und Leipzig for their participation. We also thank David Petroff for assistance with the manuscript.

\section{Funding Sources}

The study was supported by an unrestricted grant from Novartis, the Deutsche Forschungsgemeinschaft (KO 3512/1-1), and the "Roland Ernst Stiftung".

\section{References}

1. Berenson GS, Srinivasan SR, Bao W, Newman WP III, Tracy RE, Wattigney WA. Association between multiple cardiovascular risk factors and atherosclerosis in children and young adults: The Bogalusa Heart Study. N Engl J Med 1998; 338: 1650-1656.

2. Mahmud FH, Earing MG, Lee RA, Lteif AN, Driscoll DJ, Lerman A. Altered endothelial function in asymptomatic male adolescents with type 1 diabetes. Congenit Heart Dis 2006; 1: $98-103$.

3. Haller MJ, Stein J, Shuster J, Theriaque D, Silverstein J, Schatz DA, et al. Peripheral artery tonometry demonstrates altered endothelial function in children with type 1 diabetes. Pediatr Diabetes 2007; 8: 193-198.

4. Urbina EM, Williams RV, Alpert BS, Collins RT, Daniels SR, Hayman L, et al. Noninvasive assessment of subclinical atherosclerosis in children and adolescents: Recommendations for standard assessment for clinical research: A scientific statement from the American Heart Association. Hypertension 2009; 54: 919-950.

5. Halcox JP, Deanfield JE. Childhood origins of endothelial dysfunction. Heart 2005; 91: 1272-1274.

6. Corretti MC, Anderson TJ, Benjamin EJ, Celermajer D, Charbonneau F, Creager MA, et al. Guidelines for the ultrasound assessment of endothelial-dependent flow-mediated vasodilation of the brachial artery: A report of the International Brachial Artery Reactivity Task Force. J Am Coll Cardiol 2002; 39: 257 265.

7. Bonetti PO, Pumper GM, Higano ST, Holmes DR Jr, Kuvin JT, Lerman A. Noninvasive identification of patients with early coronary atherosclerosis by assessment of digital reactive hyperemia. J Am Coll Cardiol 2004; 44: 2137-2141.

8. Kuvin JT, Mammen A, Mooney P, Alsheikh-Ali AA, Karas RH. Assessment of peripheral vascular endothelial function in the ambulatory setting. Vasc Med 2007; 12: 13-16.

9. Singh TP, Groehn H, Kazmers A. Vascular function and carotid intimal-medial thickness in children with insulin-dependent diabetes mellitus. J Am Coll Cardiol 2003; 41: 661-665.

10. Muller UM, Walther C, Adams V, Mende M, Adam J, Fikenzer $\mathrm{K}$, et al. Long term impact of one daily unit of physical exercise at school on cardiovascular risk factors in school children. Eur $J$ Prev Cardiol 2016; 23: 1444-1452.

11. Walther C, Gaede L, Adams V, Gelbrich G, Leichtle A, Erbs S, et al. Effect of increased exercise in school children on physical fitness and endothelial progenitor cells: A prospective randomized trial. Circulation 2009; 120: 2251-2259.

12. Faizi AK, Kornmo DW, Agewall S. Evaluation of endothelial function using finger plethysmography. Clin Physiol Funct Imaging 2009; 29: 372-375.

13. Kuvin JT, Patel AR, Sliney KA, Pandian NG, Sheffy J, Schnall $\mathrm{RP}$, et al. Assessment of peripheral vascular endothelial function with finger arterial pulse wave amplitude. Am Heart J 2003; 146: $168-174$. 
14. Kromeyer-Hauschild K, Wabitzsch M, Kunze D, Geller F, Geiß $\mathrm{HC}$, Hesse V, et al. Perzentile für den body-mass-index für das Kindes- und Jugendalter unter Heranziehung verschiedener deutscher Stichproben. Monatsschr Kinderheilk 2001; 149: 807 818 (in German).

15. Bates D, Maechler M, Bolker B, Walker S. Fitting linear mixedeffects models using lme4. J Stat Software 2015; 67: 1-48.

16. Stasinopoulos D, Rigby R. Generalized additive models for location scale and shape (GAMLSS) in R. J Stat Software 2007; 23: $1-46$.

17. Celermajer DS, Sorensen KE, Gooch VM, Spiegelhalter DJ, Miller OI, Sullivan ID, et al. Non-invasive detection of endothelial dysfunction in children and adults at risk of atherosclerosis. Lancet 1992; 340: 1111-1115.

18. Walther C, Mende M, Gaede L, Muller U, Machalica K, Schuler G. Effects of daily physical exercise at school on cardiovascular risk--results of a 2-year cluster-randomized study. Dtsch Med Wochenschr 2011; 136: 2348-2354 (in German).

19. Kelly AS, Marlatt KL, Steinberger J, Dengel DR. Younger age is associated with lower reactive hyperemic index but not lower flow-mediated dilation among children and adolescents. Atherosclerosis 2014; 234: 410-414.

20. Bhangoo A, Sinha S, Rosenbaum M, Shelov S, Ten S. Endothelial function as measured by peripheral arterial tonometry increases during pubertal advancement. Horm Res Paediatr 2011; 76: $226-233$.

21. Radtke T, Khattab K, Eser P, Kriemler S, Saner H, Wilhelm M Puberty and microvascular function in healthy children and adolescents. J Pediatr 2012; 161: 887-891.

22. Donald AE, Charakida M, Cole TJ, Friberg P, Chowienczyk PJ, Millasseau SC, et al. Non-invasive assessment of endothelial function: Which technique? J Am Coll Cardiol 2006; 48: 18461850.

23. Juonala M, Magnussen CG, Venn A, Gall S, Kahonen M, Laitinen T, et al. Parental smoking in childhood and brachial artery flow-mediated dilatation in young adults: The Cardiovascular Risk in Young Finns study and the Childhood Determinants of Adult Health study. Arterioscler Thromb Vasc Biol 2012; 32: 1024-1031.

24. Martin WH III, Ogawa T, Kohrt WM, Malley MT, Korte E, Kieffer PS, et al. Effects of aging, gender, and physical training on peripheral vascular function. Circulation 1991; 84: 654-664.

25. Hopkins ND, Dengel DR, Stratton G, Kelly AS, Steinberger J, Zavala $\mathrm{H}$, et al. Age and sex relationship with flow-mediated dilation in healthy children and adolescents. J Appl Physiol 2015; 119: $926-933$.

26. Juonala M, Kahonen M, Laitinen T, Hutri-Kahonen N, Jokinen E, Taittonen L, et al. Effect of age and sex on carotid intimamedia thickness, elasticity and brachial endothelial function in healthy adults: The cardiovascular risk in Young Finns Study. Eur Heart J 2008; 29: 1198 -1206.

27. Litwin M, Trelewicz J, Wawer Z, Antoniewicz J, Wierzbicka A,
Rajszys $\mathrm{P}$, et al. Intima-media thickness and arterial elasticity in hypertensive children: Controlled study. Pediatr Nephrol 2004; 19: $767-774$.

28. Woo KS, Chook P, Yu CW, Sung RY, Qiao M, Leung SS, et al Overweight in children is associated with arterial endothelial dysfunction and intima-media thickening. Int J Obes Relat Metab Disord 2004; 28: 852-857.

29. Jarvisalo MJ, Putto-Laurila A, Jartti L, Lehtimaki T, Solakivi T, Ronnemaa $\mathrm{T}$, et al. Carotid artery intima-media thickness in children with type 1 diabetes. Diabetes 2002; 51: 493-498.

30. Osika W, Dangardt F, Montgomery SM, Volkmann R, Gan LM, Friberg P. Sex differences in peripheral artery intima, media and intima media thickness in children and adolescents. Atherosclerosis 2009; 203: $172-177$.

31. Nava E, Llorens $\mathrm{S}$. The paracrine control of vascular motion: A historical perspective. Pharmacol Res 2016; 113: 125-145.

32. Stoner L, Erickson ML, Young JM, Fryer S, Sabatier MJ, Faulkner J, et al. There's more to flow-mediated dilation than nitric oxide. J Atheroscler Thromb 2012; 19: 589-600.

33. Hwa JJ, Ghibaudi L, Williams P, Chatterjee M. Comparison of acetylcholine-dependent relaxation in large and small arteries of rat mesenteric vascular bed. Am J Physiol 1994; 266: H952H958.

34. Shimokawa H, Yasutake H, Fujii K, Owada MK, Nakaike R, Fukumoto $\mathrm{Y}$, et al. The importance of the hyperpolarizing mechanism increases as the vessel size decreases in endotheliumdependent relaxations in rat mesenteric circulation. $J$ Cardiovasc Pharmacol 1996; 28: 703-711.

35. Mehrke G, Pohl U, Daut J. Effects of vasoactive agonists on the membrane potential of cultured bovine aortic and guinea-pig coronary endothelium. J Physiol 1991; 439: 277-299.

36. Hedetoft M, Olsen NV. Evaluation of endothelial function by peripheral arterial tonometry and relation with the nitric oxide pathway. Nitric Oxide 2014; 42: 1-8.

37. Ostrem JD, Evanoff N, Kelly AS, Dengel DR. Presence of a high-flow-mediated constriction phenomenon prior to flowmediated dilation in normal weight, overweight, and obese children and adolescents. $J$ Clin Ultrasound 2015; 43: 495-501.

38. Muller U, Matsuo Y, Lauber M, Walther C, Oberbach A, Schuler G, et al. Correlation between endothelial function measured by finger plethysmography in children and HDLmediated eNOS activation: Apreliminary study. Metabolism 2013; 62: $634-637$.

\section{Supplementary Files}

\section{Supplementary File 1}

Table S1. Percentiles of RHI by age in half-yearly decrements in 10-17 year-old schoolchildren

Please find supplementary file(s);

http://dx.doi.org/10.1253/circj.CJ-16-0994 